\title{
Uso do alimento por duas espécies simpátricas de Moenkhausia (Characiformes, Characidae) em um riacho da Região Centro-Oeste do Brasil
}

\author{
Raffael M. Tófoli ${ }^{1}$, Norma S. Hahn ${ }^{1,2}$, Gustavo H. Z. Alves ${ }^{1}$ \& Gisele C. Novakowski ${ }^{1,3}$ \\ 1. Programa de Pós-graduação em Ecologia de Ambientes Aquáticos Continentais, Universidade Estadual de Maringá, Av. Colombo, 5790, \\ 87020-900 Maringá, PR, Brasil. (raffaeltofoli@ hotmail.com) \\ 2. Núcleo de Pesquisas em Limnologia, Ictiologia e Aquicultura, Universidade Estadual de Maringá, Av. Colombo, 5790, 87020-900 \\ Maringá, PR, Brasil. \\ 3. Unidade de Ensino Superior Ingá, Av. Colombo, 9727 KM 130, Bloco D, sala 13, 87070-810 Maringá, PR, Brasil.
}

\begin{abstract}
Food used by two sympatric species of Moenkhausia (Characiformes, Characidae), in a stream of centerwestern Brazil. This study evaluated the seasonality in the diet of Moenkhausia dichroura (Kner, 1858) and M. sanctaefilomenae (Steindachner, 1907), sympatric species of the Cancela stream, state of Mato Grosso, Brazil. Regardless of the seasonal period, allochthonous food source predominated in the diet of both species ( $>50 \%$ of all items consumed) and among these terrestrial insects were the dominant resource. However, aquatic insects were important in the diet of $M$. dichroura as well. Hymenoptera (Formicidae) was the dominant item to both species, thus they were characterized as terrestrial insectivorous. The restricted diet of these species is confirmed by the trophic niche breadth, whose values were in general low: $\mathrm{Ba}=0.26$ to $M$. dichroura in both periods and $\mathrm{Ba}=0.41$ and 0.38 in the dry and rainy period, respectively, to $M$. sanctaefilomenae. The feeding overlap was high in the rainy period $\left(\mathrm{O}_{\mathrm{jk}}=0.75\right)$ and intermediate in the dry period $\left(\mathrm{O}_{\mathrm{jk}}=0.41\right)$, showing that the food partitioning between species was larger in this period.
\end{abstract}

KEYWORDS. Diet, Moenkhausia dichroura, M. sanctaefilomenae, coexistence.

RESUMO. Esse estudo avaliou sazonalmente a dieta de Moenkhausia dichroura (Kner, 1858) e M. sanctaefilomenae (Steindachner, 1907), coletadas em simpatria no riacho Cancela, Mato Grosso, Brasil. Independente do período hidrológico, alimentos de origem alóctone predominaram na dieta de ambas as espécies ( $>50 \%$ do total de itens consumidos), sendo insetos terrestres o recurso principal, embora, para $M$. dichroura insetos aquáticos tenham contribuído na dieta também. Hymenoptera (Formicidae) foi o alimento mais consumido, sendo ambas as espécies caracterizadas como insetívoras terrestres. A dieta restrita dessas espécies é confirmada pelos baixos valores de amplitude de nicho trófico: $\mathrm{Ba}=0,26$ para $M$. dichroura em ambos os períodos e $\mathrm{Ba}=0,41$ no período de seca e 0,38 no período de chuva, para M. sanctaefilomenae. A sobreposição alimentar foi elevada no período de chuva $\left(\mathrm{O}_{\mathrm{jk}}=0,75\right)$ e apresentou valor intermediário no período de seca $\left(\mathrm{O}_{\mathrm{jk}}=0,41\right)$, evidenciando maior partilha do alimento entre as espécies neste período.

PALAVRAS-CHAVE. Dieta, Moenkhausia dichroura, M. sanctaefilomenae, coexistência.

A partilha dos recursos alimentares entre espécies coexistentes tem sido descrita como mais importante que a partilha de hábitats em ambientes aquáticos (SHOENER, 1974; Ross, 1986), sendo vista como um dos processos responsáveis na estruturação de populações e comunidades. Esse processo pode ocorrer em dois níveis, intraespecífico e interespecífico, sendo que neste último caso as espécies podem ser fillogeneticamente próximas ou não (GERKING, 1994). Espécies filogeneticamente relacionadas tendem a ser ecologicamente mais semelhantes em muitos aspectos. Consequentemente, a coexistência entre elas é difícil de explicar (MoL, 1995). Entretanto, CHASE \& LEIBOLD (2003) argumentam que, em pequenas escalas, espécies relacionadas que coexistem em comunidades geralmente exibem algum tipo de diferenciação (temporal ou espacial) na ocupação de micro-hábitats, na dieta ou em outros fatores. Nesse sentido, dados sobre alimentação subsidiam os fatores que segregam espécies simpátricas.

Moenkhausia dichroura (Kner, 1858), conhecida popularmente como lambari ou lambari-corintiano, e Moenkhausia sanctaefilomenae (Steindachner, 1907) (lambari olho-de-fogo, pequira, maconherinho) pertencem à família Characidae, subfamília Tetragonopterinae, a qual representa um aglomerado polifilético incertae sedis dentro da família (BuCKUP, 1999; BuCKUP, 2003; LiMA et al., 2003).
Ambas as espécies foram encontradas em simpatria no riacho Cancela, localizado na bacia dos rios Manso/Cuiabá, Mato Grosso, corroborando a informação de BuCKUP (1999) sobre o predomínio de Moenkhausia Eigenmann, 1903 em riachos localizados mais ao norte do país.

A oportunidade de encontrar espécies congêneres em simpatria e a importância de estudar riachos (devido às peculiaridades desse tipo de ambiente e de sua ictiofauna) podem fornecer informações singulares para questões ecológicas, tais como competição e partilha do alimento.

Este estudo avaliou a dieta de duas espécies simpátricas de Moenkhausia, objetivando responder as seguintes perguntas: existe partilha de alimento entre $M$. dichroura e $M$. sanctaefilomenae? e a sazonalidade interfere na utilização dos recursos alimentares?

\section{MATERIAL E MÉTODOS}

Área de estudo. Os peixes foram amostrados no riacho Cancela, tributário do rio Cuiabá, constituinte da bacia dos rios Manso/Cuiabá, Mato Grosso (Fig. 1). No trecho amostrado o riacho apresentou cerca de $3 \mathrm{~m}$ de largura, profundidade máxima de $40 \mathrm{~cm}$, fundo com predominância de cascalho e cobertura abundante de material alóctone, principalmente folhas em decomposição e pequenos galhos. O riacho apresenta 
vegetação ciliar impactada por ação antrópica, com algumas áreas ainda preservadas, encostas superiores dominadas por pastagens para criação de gado e alguns rebanhos de cabras, além de áreas com culturas de subsistência (milho e mandioca).

Amostragem e análise dos dados. As amostragens foram realizadas mensalmente, de março de 2003 a fevereiro de 2004. A distinção dos períodos de seca e chuva foi feita com base na análise das variações dos níveis hidrológicos locais (fornecidos pela ANA Agência Nacional de Águas). Foram considerados como meses de chuva aqueles em que ocorreram chuvas em até 10 dias antes da coleta. Desta forma, o período entre abril e setembro foi considerado como período de seca, enquanto que o período entre outubro e março foi considerado como período de chuva. Os peixes foram coletados empregando-se a pesca elétrica, que consiste em produzir um campo elétrico na água, passando uma corrente entre dois eletrodos submersos (UIEDA \& CAstro, 1999). O equipamento funciona com auxílio de um gerador de $220 \mathrm{~V}$, acoplado a um transformador de alta voltagem com conversor de corrente $(1,5 \mathrm{KW}, 200$, 300 e $500 \mathrm{~V}, 1 \mathrm{~A}$ ) equipado com um cabo com saída para dois puçás e aro metálico (cátodo e ânodo). O limite inferior do trecho amostrado foi bloqueado com uma rede de malha de $1 \mathrm{~cm}$ entre nós opostos, no sentido jusantemontante.

Exemplares testemunhos dos peixes capturados e utilizados nesse estudo foram depositados na coleção de peixes do Núcleo de Pesquisas em Limnologia, Ictiologia e Aqüicultura (Nupélia/UEM): $M$. dichroura (NUP 4096) e M. sanctaefilomenae (NUP 929).

Cada exemplar foi medido (comprimento padrão, $\mathrm{cm}$ ), pesado (peso total, g) e eviscerado, sendo os estômagos com alimento fixados em formol $4 \%$ para posterior análise em laboratório.

A dieta de cada espécie foi avaliada separadamente para os períodos de seca e chuva, de acordo com: a origem do alimento (autóctone, alóctone e indeterminada); os

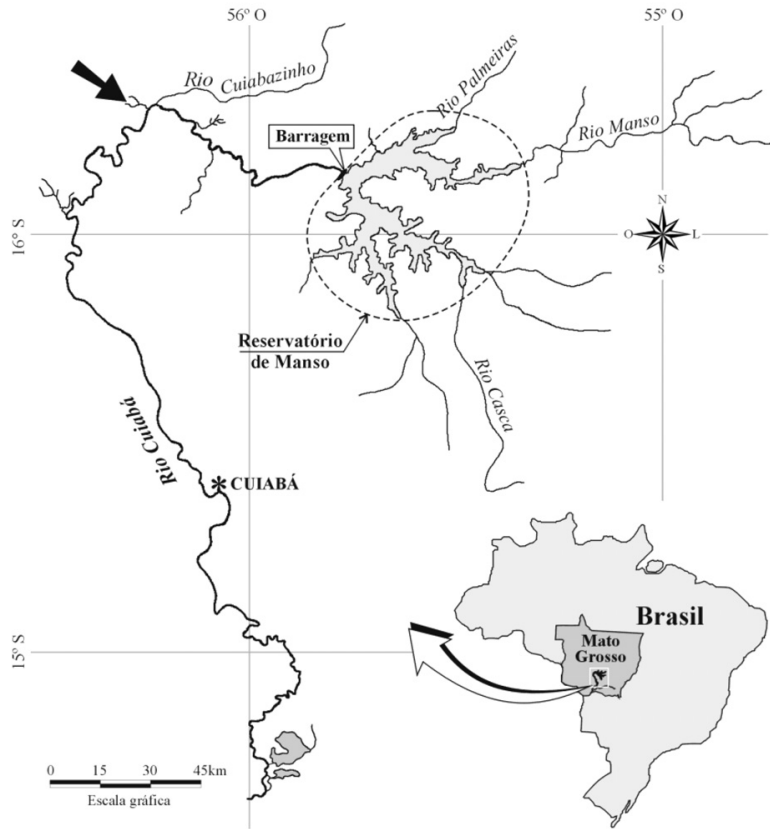

Figura 1. Mapa da bacia dos rios Manso/Cuiabá, Mato Grosso, Brasil. O riacho Cancela está indicado pela seta escura recursos alimentares (alimento identificado em nível de grandes grupos) e os itens alimentares (alimento identificado em nível taxonômico, o mais inferior possível). Foram utilizados os métodos de frequência volumétrica $(\% \mathrm{~V})$ e/ou de ocorrência $(\% \mathrm{~F})$, dependendo da análise (Hyslop, 1980). No primeiro método, registrase o volume de cada item alimentar, obtendo-se a porcentagem em relação ao volume total dos conteúdos estomacais. $\mathrm{O}$ volume de cada item foi obtido $\mathrm{em}^{\mathrm{mm}^{3}}$ através de placa milimetrada e posteriormente transformado em ml (Hellawel \& ABel, 1971). No segundo método, é registrado o número de peixes em que cada item ocorre, calculando-se a porcentagem em relação ao total de estômagos com alimento.

A amplitude de nicho trófico (amplitude da dieta) foi calculada para os períodos de seca e chuva para cada espécie, usando o índice padronizado de Levins (HuRLBERT, 1978). Esse índice varia de 0, quando a espécie consumiu somente um tipo de alimento, a 1, quando a espécie consumiu de forma similar vários tipos de alimento. $\mathrm{O}$ índice é dado pela fórmula:

$$
\mathrm{Ba}=\left[\left(\sum_{j} P_{i j}^{2}\right)^{-1}-1\right](\mathrm{n}-1)^{-1}
$$

onde, $\mathrm{Ba}=$ amplitude do nicho trófico padronizada; $P_{i j}=$ proporção do item alimentar $j$ na dieta da espécie $i ; \mathrm{n}=$ número total de itens alimentares.

A sobreposição alimentar foi estimada considerando-se os períodos de seca e chuva para cada espécie, de acordo com o índice de Pianka (1973), que varia de 0 (nenhuma sobreposição) a 1 (sobreposição total) e é dado pela fórmula:

$$
O_{j k}=\frac{\sum_{i}^{n} p_{i j} p_{i k}}{\sqrt{\sum_{i}^{n} p_{i j}{ }^{2} \sum_{i}^{n} p_{i k}{ }^{2}}}
$$

onde, $O_{j k}=$ medida de sobreposição alimentar de Pianka entre a espécie $j$ e a espécie $k ; p_{i j}=$ proporção do item alimentar $i$ no total de itens utilizados pela espécie $j ; p_{i k}=$ proporção do item alimentar $i$ no total de itens utilizados pela espécie $k, n=$ número total de itens alimentares. Os resultados da sobreposição interespecífica foram arbitrariamente considerados: alto $(>0,6)$, intermediário $(0,4-0,6)$ ou baixo $(<0,4)$ (GROSSMAN, 1986).

Para avaliar se o padrão de sobreposição observado difere do padrão gerado ao acaso, a matriz original dos dados foi aleatorizada. Utilizando um modelo nulo, as proporções de volume dos itens alimentares observados para cada espécie foram randomizadas 10.000 vezes e, para cada randomização, um índice de Pianka foi calculado. Dessa forma, a significância estatística foi determinada através da comparação da sobreposição observada com a distribuição de valores nulos, considerando significância ao nível de $\alpha<0,05$. Para essa análise foi utilizado o programa EcoSim (Gotelli \& ENTSMINGER, 2006).

\section{RESULTADOS}

Foram analisados 124 conteúdos estomacais de $M$. dichoura e 91 de $M$. sanctaefilomenae. Alimentos de origem alóctone predominaram na dieta de ambas as espécies, independentemente do período hidrológico considerado, enquanto que os de origem autóctone representaram a segunda categoria mais importante na seca. Para M. sanctaefilomenae, recursos indeterminados, 
representados por detritos, foram também relevantes neste mesmo período (Fig. 2).

Insetos terrestres foram dominantes na dieta de ambas as espécies, principalmente no período de chuva. Entretanto, para M. dichroura, insetos aquáticos também tiveram importante participação em ambos os períodos (Figs 3-6), principalmente em ocorrência ( $\geq 70 \%)$.

Dentre os insetos terrestres, Hymenoptera (Formicidae) foi o alimento mais consumido (ocorrência x volume) por ambas as espécies de modo geral, especialmente no período de chuva. Entretanto, na dieta de $M$. dichroura, Coleoptera e Diptera tiveram participação relevante também. Com relação aos insetos aquáticos, destacaram-se Chironomidae, Ephemeroptera, outros Diptera e outros insetos aquáticos, em ocorrência e/ ou volume, dependendo do período considerado (Tab. I).

O consumo elevado de insetos (tanto formas adultas quanto imaturas) sugere uma dieta restrita para ambas as espécies, o que é comprovado pelos baixos valores de amplitude de nicho trófico $(<0,6)$ : $M$. dichroura ( $\mathrm{Ba}=0,26$ em ambos os períodos) e M. sanctaefilomenae ( $\mathrm{Ba}=0,41$ no período de seca e $\mathrm{Ba}=0,38$ no período de chuva) (Fig. 7).

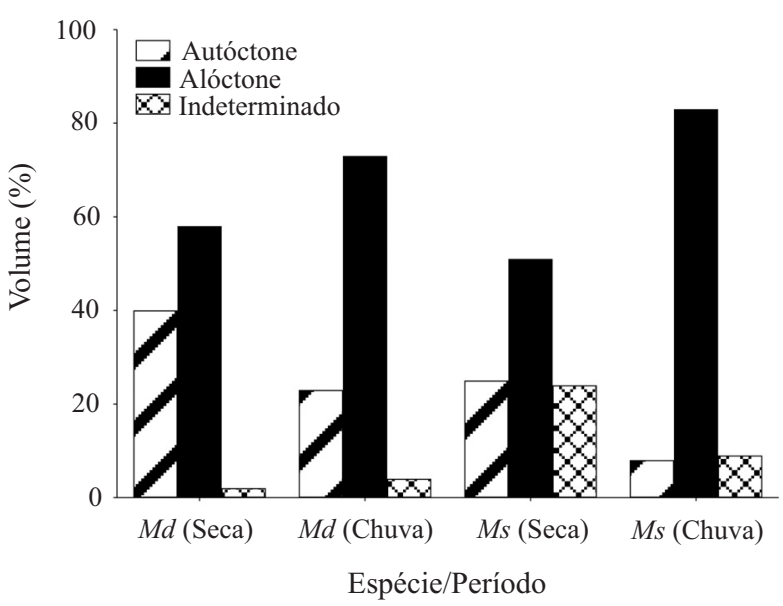

Figura 2. Frequência volumétrica (V\%) dos recursos de origens autóctone, alóctone e indeterminada, consumidos por Moenkhausia dichroura (Kner, 1858) - Md e por M. sanctaefilomenae (Steindachner, 1907) - Ms, no riacho Cancela, bacia dos rios Manso/ Cuiabá, Mato Grosso, Brasil, de março de 2003 a fevereiro de 2004.

Tabela I. Composição da dieta de $M$. dichroura (Kner, 1858) e M. sanctaefilomenae (Steindachner, 1907) no riacho Cancela, bacia dos rios Manso/Cuiabá, Mato Grosso, Brasil, de março de 2003 a fevereiro de 2004 (\%F, frequência de ocorrência dos itens alimentares; OIA, outros insetos aquáticos; OIT, outros insetos terrestres; \%V, frequência volumétrica dos itens alimentares).

\begin{tabular}{|c|c|c|c|c|c|c|c|c|}
\hline & \multicolumn{4}{|c|}{ M. dichroura } & \multicolumn{4}{|c|}{ M. sanctaefilomenae } \\
\hline & \multicolumn{2}{|c|}{ Seca } & \multicolumn{2}{|c|}{ Chuva } & \multicolumn{2}{|c|}{ Seca } & \multicolumn{2}{|c|}{ Chuva } \\
\hline & $\% \mathrm{~V}$ & $\% \mathrm{~F}$ & $\% \mathrm{~V}$ & $\% \mathrm{~F}$ & $\% \mathrm{~V}$ & $\% \mathrm{~F}$ & $\% \mathrm{~V}$ & $\% \mathrm{~F}$ \\
\hline \multicolumn{9}{|l|}{ AUTÓCTONE } \\
\hline Chironomidae & 3,29 & 34,41 & 0,27 & 12,90 & 0,34 & 12,90 & 0,20 & 2,22 \\
\hline Outros Diptera & 5,53 & 27,96 & 8,16 & 41,94 & 6,45 & 11,11 & 2,15 & 22,58 \\
\hline Ephemeroptera & 25,30 & 33,33 & 7,77 & 19,35 & & & & \\
\hline OIA & 3,00 & 12,90 & 5,37 & 32,26 & 2,43 & 10,00 & 2,98 & 25,81 \\
\hline Nematoda & 0,11 & 3,23 & 0,04 & 3,23 & & & & \\
\hline Annelida & 1,22 & 3,23 & & & & & & \\
\hline Tecameba & 0,07 & 3,23 & 0,08 & 6,45 & & & & \\
\hline Escamas & 0,47 & 10,75 & 0,43 & 12,90 & 0,16 & 3,33 & 0,08 & 3,23 \\
\hline Peixe & & & & & 16,28 & 4,44 & 2,44 & 3,23 \\
\hline Algas & 0,76 & 12,90 & 1,37 & 22,58 & 0,26 & 5,56 & 0,02 & 6,45 \\
\hline \multicolumn{9}{|l|}{ ALÓCTONE } \\
\hline Hymenoptera & 20,78 & 54,84 & 30,01 & 87,10 & 14,54 & 42,22 & 28,20 & 93,55 \\
\hline Hemiptera & 1,49 & 9,68 & 0,59 & 3,23 & 1,79 & 11,11 & 3,95 & 19,35 \\
\hline Homoptera & 0,78 & 2,15 & 0,39 & 3,23 & 4,37 & 8,89 & 5,37 & 19,35 \\
\hline Coleoptera & 9,06 & 31,18 & 4,2 & 35,48 & 2,32 & 18,89 & 17,46 & 18,89 \\
\hline Thysanoptera & 0,82 & 9,68 & 0,59 & 9,68 & & & & \\
\hline Isoptera & & & 9,06 & 22,58 & & & & \\
\hline Diptera & 11,13 & 30,11 & 8,24 & 51,61 & & & & \\
\hline Trichoptera & 3,66 & 7,53 & & & 12,06 & 20,00 & 10,44 & 35,48 \\
\hline OIT & 7,95 & 30,11 & 13,53 & 29,03 & 18 & 36,67 & 8,78 & 48,39 \\
\hline Araneae & 1,84 & 7,53 & 5,18 & 12,9 & 0,96 & 8,89 & 0,83 & 9,68 \\
\hline Pseudoscorpiones & 0,44 & 3,23 & & & & & & \\
\hline Chilopoda & & & 0,59 & 3,23 & & & & \\
\hline Diplopoda & & & & & & & 7,32 & 9,68 \\
\hline Acarina & & & 0,39 & 3,23 & & & & \\
\hline \multicolumn{9}{|l|}{ INDETERMINADO } \\
\hline Vegetal & 0,78 & 9,68 & 2,35 & 25,81 & 24,9 & 26,67 & 7,17 & 26,67 \\
\hline Detrito & 1,53 & 10,75 & 1,37 & 22,58 & 0,19 & 3,33 & 1,76 & 6,45 \\
\hline $\mathrm{N}^{\mathrm{o}}$ de estômagos & \multicolumn{2}{|c|}{93} & \multicolumn{2}{|c|}{31} & \multicolumn{2}{|c|}{60} & \multicolumn{2}{|c|}{31} \\
\hline
\end{tabular}



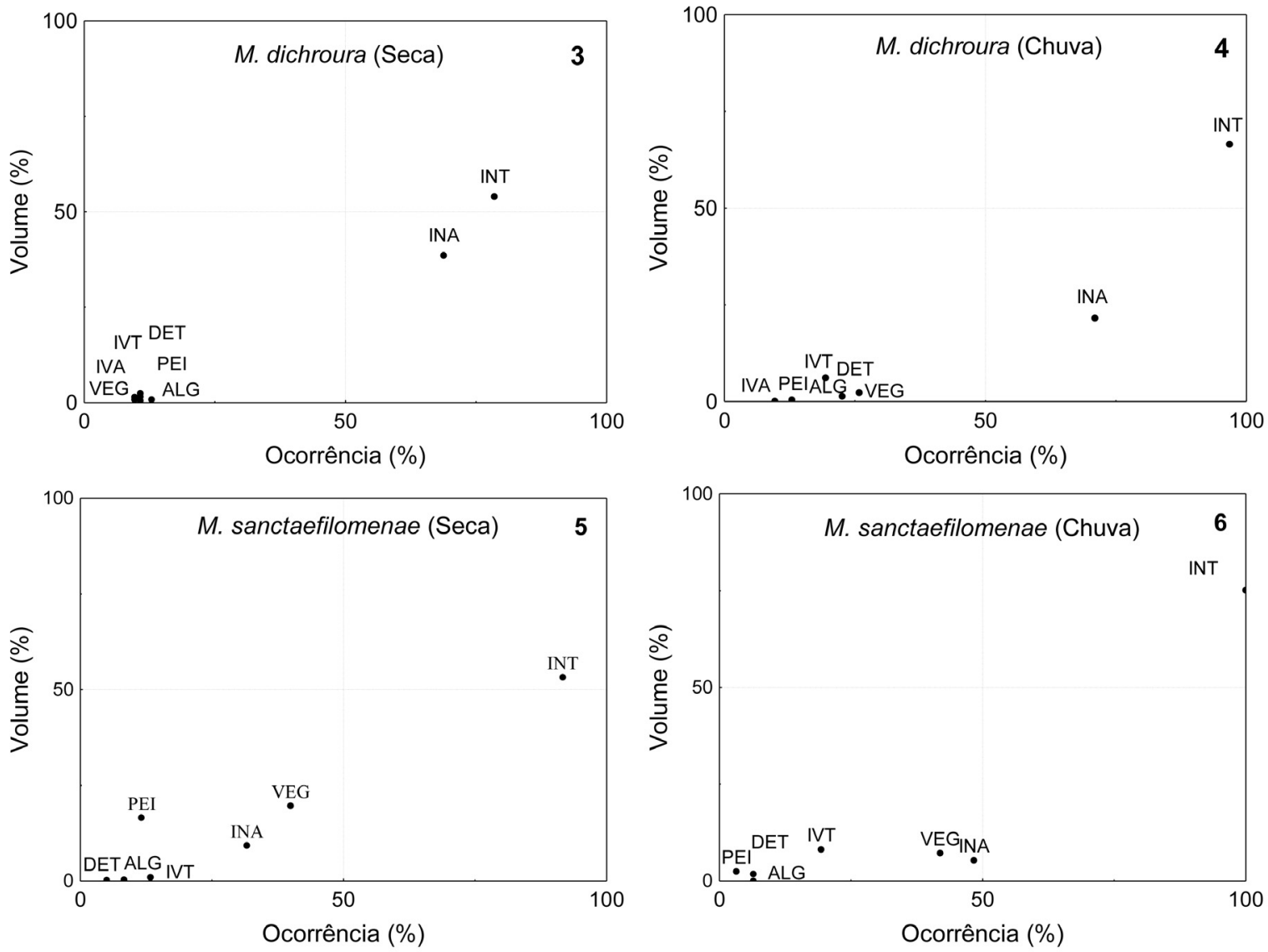

Figuras 3-6. Recursos alimentares consumidos por Moenkhausia dichroura (Kner, 1858) e M. sanctaefilomenae (Steindachner, 1907) no riacho Cancela, bacia dos rios Manso/Cuiabá, Mato Grosso, Brasil, de março de 2003 a fevereiro de 2004: 3, M. dichroura durante o período de seca; 4, período de chuva; 5, M. sanctaefilomenae durante o período de seca; 6, período de chuva (ALG, algas; DET, detrito/sedimento; INA, insetos aquáticos; INT, insetos terrestres; IVA, invertebrados aquáticos; IVT, invertebrados terrestres; PEI, peixes; VEG, vegetal).

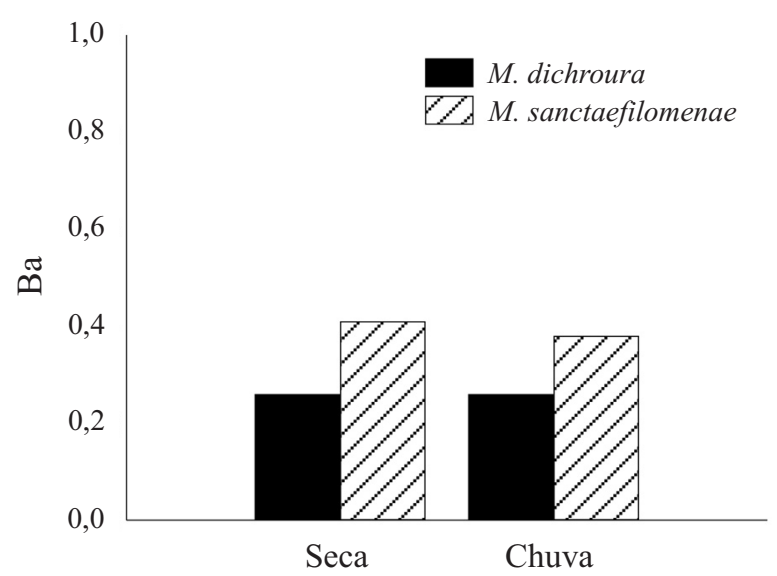

Figura 7. Amplitude de nicho trófico (Ba) de Moenkhausia dichroura (Kner, 1858) e de M. sanctaefilomenae (Steindachner, 1907) no riacho Cancela, bacia dos rios Manso/Cuiabá, Mato Grosso, Brasil, de março de 2003 a fevereiro de 2004.

Os valores de sobreposição alimentar interespecífica variaram de intermediário no período de $\operatorname{seca}\left(\mathrm{O}_{\mathrm{jk}}=0,41\right)$ (média simulada $\left.=0,32 ; \mathrm{p}=0,23\right)$ a elevados no período de chuva $\left(\mathrm{O}_{\mathrm{jk}}=0,75\right)$, sendo este último valor significantemente maior do que o esperado (média simulada $=0,28 ; p=0,02$ ). Por conseguinte, os valores observados não poderiam ser originados ao acaso e refletem, portanto, processos biológicos.

\section{DISCUSSÃO}

O consumo expressivo de alimento de origem alóctone constatado para as duas espécies de Moenkhausia é bem documentado na literatura para peixes de riachos tropicais (SABINO \& CASTRO, 1990; Henry et al., 1994; SABInO \& ZuAnON, 1998; Esteves \& Aranha, 1999; CAStro, 1999; Graciolli et al., 2003). O sombreamento produzido pela vegetação arbórea limita a produção primária e os peixes se tornam dependentes dos recursos provenientes das encostas (LoweMcConnell, 1999), sendo esse tipo de alimento considerado como o principal recurso energético para habitantes destes ambientes (STEWART \& DAVIES, 1990). No riacho Cancela, a elevada contribuição de recursos alóctones na dieta das espécies de Moenkhausia provavelmente está associada à mata ripária moderadamente preservada. Porém, o incremento no consumo deste tipo de recurso no período chuvoso, para ambas as espécies, certamente está associado ao carreamento de invertebrados terrestres pela chuva e/ou pelo vento, bem como ao alagamento das margens, que amplia a área de forrageamento. Teoricamente, é nesta época que ocorre maior disponibilidade de invertebrados terrestres para os peixes desses ambientes, devido à maior produtividade geral e à expansão no curso de água para áreas marginais (LowE-McCoNNELL, 1999). 
Insetos terrestres foram predominantes nos conteúdos estomacais ao longo de todo o período de estudo, fato amplamente estabelecido para a ictiofauna de riachos (ANGERMEIER \& KARR, 1983; GARMAN, 1991; REzende \& MAZzoni, 2006). Apesar de vários estudos sobre a alimentação de espécies de Characidae indicarem forte influência sazonal na dieta (ARANHA et al., 2000; Esteves \& Pinto-Lobo, 2001; MAZzoni \& ReZende, 2003), para as espécies de Moenkhausia esta variável teve pouca influência, o que sugere que estes insetos estiveram disponíveis durante todo o ano. Dessa forma, ambas as espécies apresentaram hábito alimentar insetívoro, com tendência à captura de insetos terrestres, provavelmente na superfície da água. Este fato é corroborado pelo estudo de CASATTI (2002), no córrego São Carlos (SP), onde M. sanctaefilomenae praticou, em $95 \%$ dos registros, a cata de itens arrastados pela corrente (driftfeeding, GRANT \& NOAKES, 1987). Estudos referentes à dieta de $M$. dichroura mostram que, dependendo do ambiente e da sazonalidade, a dieta é composta por zooplâncton e insetos (Poullly et al., 2003; REJAs et al., 2005). Na planície de inundação do rio Mamoré, Bolívia, Poullly et al. (2004) verificaram predomínio de insetos aquáticos e terrestres na dieta desta espécie, enquanto que Silva \& Hahn (2009) relatam o consumo expressivo de zooplâncton no início da formação do reservatório de Manso (Mato Grosso).

Hymenoptera (Formicidae) foi um item altamente expressivo para ambas as espécies independente do período hidrológico, mostrando-se especialmente abundante nos conteúdos estomacais no período de chuva. Borba et al. (2008) registraram que, dentre os insetos terrestres consumidos por Astyanax asuncionensis Géry, 1972 (também no riacho Cancela), Hymenoptera esteve entre os principais itens. Dessa forma, é possível inferir que o consumo destes insetos esteve associado à sua elevada abundância e disponibilidade no período considerado. Uma provável causa da disponibilidade seria a queda de indivíduos provenientes do dossel da mata pela ação da chuva e do vento, uma vez que as formigas foram consumidas mesmo durante o período da seca. A mata ripária, sem sinais severos de degradação, pode ter sido importante na oferta destes e de outros insetos terrestres, também constatados na dieta das espécies. É relatado que dentre os artrópodes, as formigas se destacam em número e biomassa na ocupação de copas de florestas tropicais (HARADA \& ADIS, 1998; SANTOs et al., 2003; BATTIROLA et al., 2007).

Por conseguinte, ambas as espécies apresentaram dieta restrita, a qual foi confirmada pelos baixos valores de amplitude de nicho trófico $(<0,6)$, podendo ser consideradas especialistas (sensu Pouilly et al., 2004). Porém, para as espécies contempladas neste estudo, é mais prudente denominá-las de especialistas pontuais, pois uma dieta restrita nem sempre indica especialização. Uma espécie pode ser induzida a consumir uma determinada fonte de alimento que se encontra temporariamente abundante no ambiente e este provavelmente seja o caso dos Hymenoptera no riacho Cancela.

A sobreposição alimentar foi alta no período de chuva, principalmente devido ao elevado consumo de insetos terrestres (em especial Hymenoptera) por ambas as espécies, embora outros tipos de alimento tenham sido consumidos em pequenas proporções. Já na estação seca, as espécies apresentaram um maior grau de segregação trófica. Assim, além de insetos terrestres, $M$. dichroura passou a consumir insetos aquáticos (principalmente Chironomidae e Ephemeroptera) em proporções expressivas, enquanto que para $M$. sanctaefilomenae vegetais e também insetos aquáticos (principalmente larvas de Diptera) foram categorias importantes. Portanto, as dietas foram mais semelhantes no período de chuva, época em que supostamente ocorre maior oferta de alimento (MARÇAL-SimABUKu \& PERET, 2002). Entretanto, parece não haver um padrão sazonal determinante na tomada do alimento entre os peixes. LowE-MCCONNELL (1964) relata baixos valores de sobreposição no período de chuva, quando o alimento é abundante e os peixes podem se segregar mais facilmente. Entretanto, ZARET \& Rand (1971), Prejs \& Prejs (1987) e Winemiller (1989) registraram baixos valores de sobreposição durante a época seca. Em contrapartida, MARÇAL-SimABUKU \& PERET (2002) e NovaKowski et al. (2008) não encontraram diferenças entre períodos hidrológicos distintos.

Dessa forma, as espécies de Moenkhausia apresentaram dietas semelhantes, quando se considera que ambas consumiram os mesmos tipos de recursos alimentares, tais como, insetos e outros invertebrados terrestres e aquáticos, vegetais e detritos. Entretanto, as proporções desses recursos nos conteúdos estomacais diferiram entre os períodos hidrológicos, evidenciando maior partilha do alimento no período de seca.

Agradecimentos. Os autores agradecem ao Nupélia (Núcleo de Pesquisas em Limnologia, Ictiologia e Aquicultura) pelo apoio logístico e ao Convênio UEM/Nupélia/Furnas Centrais Elétricas pelo apoio financeiro.

\section{REFERÊNCIAS BIBLIOGRÁFICAS}

Angermeier, P. L. \& KARR, J. R. 1983. Fish communities along environmental gradients in a system of tropical streams. Environmental Biology of Fishes 9(2):117-135.

Aranha, J. M. R.; Gomes, J. H. C. \& Fogaça, F. N. O. 2000. Feeding of two species of Characidium, $C$. lanei and $C$. pterostictum (Characidiinae) in a coastal stream of atlantic forest (southern Brazil). Brazilian Archives of Biology and Technology 43(5):527-531.

Battirola, L. D.; Adis, J.; Marques, M. I. \& Silva, F. H. O. 2007. Comunidade de artrópodes associada à copa de Attalea phalerata Mart. (Arecaceae) durante o período de cheia no Pantanal de Poconé, MTt. Neotropical Entomology 36(5):640-651.

Borba, C. S.; Fugi, R.; Agostinho, A. A. \& Novakowski, G. C. 2008. Dieta de Astyanax asuncionensis (Characiformes, Characidae), em riachos da bacia do rio Cuiabá, estado do Mato Grosso. Acta Scientiarum Biological Sciences 30(1):39-45.

Buckup, P. A. 1999. Sistemática e biogeografia de peixes de riachos. In: Caramaschi, E. P.; Mazzoni, R. \& Peres-Neto, P. R. eds. Ecologia de peixes de riachos. Série Oecologia Brasiliensis. Rio de Janeiro, PPGE-UFRJ. p.19-138. 2003. Astyanax. In: Buckup, P. A. \& Menezes, N. A. eds. Catálogo dos peixes marinhos e de água doce do Brasil Disponível em: <http://www.mnrj.ufrj.br/catalogo/>. Acesso em: 15.03 .2007

Casatti, L. 2002. Alimentação dos peixes em um riacho do parque estadual Morro do Diabo, bacia do alto rio Paraná, sudeste do Brasil. Biota Neotropica 2(2):1-14.

Castro, R. M. C. 1999. Evolução da ictiofauna de riachos sulamericanos: padrões gerais e possíveis processos causais. In: 
Caramaschi, E. P.; Mazzoni, R.; Bizerril, C. R. S. F. \& PeresNeto, P. R. eds. Ecologia de peixes de riachos: estado atual e perspectivas. Série Oecologia Brasiliensis. Rio de Janeiro, PPGE-UFRJ. p.139-155.

Chase, J. M. \& Leibold, M. A. 2003. Ecological niches: linking classical and contemporary approaches. Chicago, University Chicago. 212p.

Esteves, K. E. \& Aranha, M. R. 1999. Ecologia trófica de peixes de riachos. In: Caramaschi, E. P.; Mazzoni, R. \& Peres-Neto, P R. eds. Ecologia de peixes de riachos. Série Oecologia Brasiliensis. Rio de Janeiro, PPGE-EFRJ. p.157-182.

Esteves, K. E. \& Pinto-Lobo, A. V. 2001. Feeding pattern of Salminus maxillosus (Pisces, Characidae) at Cachoeira das Emas, Mogi-Guaçu river (São Paulo state, southeastern Brazil). Brazilian Journal of Biology 61(2):267-276

Garman, G. C. 1991. Use of terrestrial arthropod prey by a stream-dwelling cyprinid fish. Environmental Biology of Fishes 30:325-331.

Gerking, S. D. 1994. Feeding ecology of fish. New York, Academic. p.416.

Gotelli, N. J. \& Entsminger, G. L. 2006. Ecosim: Null models software for ecology. Version 7. Disponível em: <http:// garyentsminger.com/ecosim.htm>. Acesso em: 20.08.2008.

Graciolli, G.; Azevedo, M. A. \& Melo, F. A. G. 2003. Comparative study of the diet of Glandulocaudinae and Tetragonopterinae (Ostariophysi, Characidae) in a small stream in southern Brazil. Studies on Neotropical Fauna and Environment 38(2): $95-110$.

Grant, J. W. A. \& Noakes, D. L. G. 1987. A simple model of optimal territory size for drift-feeding fishes. Canadian Journal of Zoology 65:270-276.

Grossman, G. D. 1986. Food resources partitioning in a rocky intertidal fish assemblage. Journal of Zoology 1:317-355.

HaRADA, A. Y. \& ADIS, J. 1998. Ants obtained from trees of a "Jacareúba" (Calophyllum brasiliense) forest plantation in Central Amazonian by canopy fogging: first results. Acta Amazônica 28:309-318.

Hellawell, J. M. \& Abel, R. 1971. A rapid volumetric method for the analysis of the food of fishes. Journal of Fish Biology 3:29-37.

Henry, R.; Uieda, V. S.; Afonso, A. A. \& Kikuchi, R. M. 1994 Input of allochthonous matter and structure of fauna in brazilian headstream. Verhandlungen der Internationale Vereinigung fur Theoretische und Angewandte Limnologie 25(3):1867-1869.

Hurlbert, S. H. 1978. The measurement of niche overlap and some relatives. Ecology 59(1):67-77.

Hyslop, E. J. 1980. Stomach contents analysis, a review of methods and their application. Journal of Fish Biology 17:411-429.

Lima, F. C. T.; Malabarba, L. R.; Buckup, P. A.; Silva, J. F. P; Vari, R. P.; Harold, A.; Benine, R.; Oyakawa, O. T.; Pavanelli, C. S.; Menezes, N. A.; Lucena, C. A. S.; Malabarba, M. C. S. L.; Lucena, Z. M. S.; Reis, R. E.; Langeani, F.; Cassati, L.; Bertaco, V. A.; Moreira, C. \& Lucinda, P. H. F. 2003. Genera incertae sedis in Characidae. In: Reis, R. E.; Kullander, S. O. \& Ferraris JR., C. J. eds. Check list of freshwater fishes of South Central America. Porto Alegre, EDIPUCRS. p.104-169.

Lowe-Mcconnell, R. H. 1964. The fishes of the Rupununi savanna district of British Guiana, South America. Journal of Linnean Society of London, Zoology 45:103-144.

1999. Estudos ecológicos em comunidades de peixes tropicais. São Paulo, EDUSP. 535p.

Marçal-Simabuku, M. A. \& Peret, A. C. 2002. Alimentação de peixes (Osteichthyes, Characiformes) em duas lagoas de uma planície de inundação brasileira da bacia do rio Paraná. Interciencia 27(6):299-305

Mazzoni, R. \& Rezende, C. F. 2003. Seasonal diet shift in Tetragonopterinae (Osteichthyes, Characidae) from Ubatiba river, RJ, Brazil. Brazilian Journal of Biology 63(1):69-74.

Mol, J. H. 1995. Ontogenetic diet shifts and diet overlap among three closely related neotropical armoured catfishes. Journal of Fish Biology 47:788-807.

Novakowski, G. C.; Hahn, N. S. \& Fugi, R. 2008. Diet seasonality and food overlap of the fish assemblage in a Pantanal pond. Neotropical Ichthyology 6(4):567-576.

Pianka, E. R. 1973. The structure of lizard communities. Annual Review of Ecology and Systematics 4:53-74

Poullly, M.; Lino, F.; Bretenoux, J. G. \& Rosales, C. C. 2003. Dietary-morphological relantionships in a fish assemblage of the Bolivian Amazonian floodplain. Journal of Fish Biology 62(5): 1137-1158.

Poullly, M.; Yunoki, T.; Rosales, C. \& Torres, L. 2004. Trophic structure of fish assemblages from Mamoré River floodplain lakes (Bolivia). Ecology of Freshwater Fish 13:245-257.

Prejs, A. \& Press, K. 1987. Feeding of tropical freshwater fishes: seasonality in resource availability and resource use. Oecologia 71:397-404

Rejas, D.; Villarpando, P. \& Carvajal, F. 2005. Variaciones estacionales em la dieta de Moenkhausia dichroura Kner (Pisces, Characidae) em una laguna de la várzea del rio Ichilo (Cochabamba-Bolivia). Revista Boliviana de Ecologia y Conservación Ambiental 17:49-54.

Rezende, C. F. \& Mazzoni, R. 2006. Contribuição da matéria autóctone e alóctone para a dieta de Bryconamericus microcephalus (Miranda-Ribeiro) (Actinopterygii, Characidae), em dois trechos de um riacho de Mata Atlântica. Revista Brasileira de Zoologia 23(1):58-63

Ross, S. T. 1986. Resource partitioning in fish assemblages: a review of field studies. Copeia 1986:352-388

Sabino, J. \& CAstro, R. M. C. 1990. Alimentação, período de atividade e distribuição espacial dos peixes de um riacho da floresta Atlântica (Sudeste do Brasil). Revista Brasileira de Biologia 50:23-36

Sabino, J. \& Zuanon, J. 1998. A stream fish assemblage in Central Amazonia: distribution, activity patterns and feeding behavior. Ichthyology Exploration of Freshwaters 8:201-210.

Santos, G. B.; Marques, M. I.; Adis, J. \& DE Musis, C. R. 2003. Artrópodos associados à copa de Attalea phalerata Mart. (Arecaceae), na região do Pantanal de Poconé-MT. Revista Brasileira de Entomologia 47:211-224.

Schoener, T. W. 1974. Resource partitioning in ecological communities. Science 185:27-38

Silva, M. R. \& HaHN, N. S. 2009. Influência da dieta sobre a abundância de Moenkhausia dichroura (Characiformes, Characidae) no reservatório de Manso, Estado de Mato Grosso. Iheringia, Série Zoologia, 99(3):324-328.

Stewart, B. A. \& Davies, B. R. 1990. Allochthonous input and retention in a small mountain stream. South Africa. Hydrobiologia 202:135-146.

Uieda, V. S. \& Castro, R. M. C. 1999. Coleta e fixação de peixes de riacho. In: Caramaschi, E. P.; Mazzoni, R. \& Peres-Neto, P R. eds. Ecologia de peixes de riachos. Série Oecologia Brasiliensis. Rio de Janeiro, PPGE-EFRJ. p.1-22.

Winemiller, K. O. 1989. Ontogenetic diet shifts and resource partitioning among piscivorous fishes in the Venezuelan Lhanos. Environmental Biology of Fishes 26:177-199.

ZARET, T. M. \& RAND, A. S. 1971. Competition in tropical stream fishes: support for the competitive exclusion principle. Ecology 52:336-342. 